\title{
Benchmark of a parallel fluid code for parametric decay instability in inhomogeneous plasmas \\ Xinfeng Sun ${ }^{1, a}$, Xiaohong Wang ${ }^{1, b}$ \\ ${ }^{1}$ State Key Laboratory of Advanced Electromagnetic Engineering and Technology, School \\ of Electrical and Electronic Engineering, Huazhong University of Science and Technology, Wuhan 430074, China \\ axinfenghappy@126.com, 'bangxh081234@126.com
}

Keywords: Parametric decay instability; Inhomogeneous plasma; Parallel fluid code;

Abstract: A nonlinear and parallel fluid code is developed to solve the full space-time domain coupled two-fluid plasma and Maxwell equations and is used to investigate the parametric decay instabilities (PDIs) of the inhomogeneous plasma. Under the laser plasma parameters of nuclear fusion, the threshold power of TPD instability measured in the simulation agree well with the analytical results. For the $X$ mode wave heating of electron cyclotron frequency in Tokamaks, the channels of upper-hybrid wave decay and Raman Scattering of electron cyclotron wave are also found.

\section{Introduction}

During the past four decades, PDIs have already been developed deeply in experiments and theories [1-11]. But there still some deficiencies exist for the large scale space problem. In recent, the authors supplied some PDIs results of ion Bernstein wave with the method of the coupled Maxwell and plasma equations in full space-time domain [9], the results indicate that, the full space-time domain simulation can offer all of linear and nonlinear physics processes and all of frequency and wave vector components. Comparing to the other methods, the full space-time domain simulation has its own advantages for the study of the PDI.

In this paper, based on the finite difference method, the coupled Maxwell equations and plasma equations are solved with full space-time solutions to recur the PDIs of laser plasma of ICF and RF heating of MCF. By this method, in fluid description limits, all the linear and nonlinear parametric decay processes of wave and plasma can be contained, and each channel can be identified from the frequency and wave vector components that obtain by simulations. Moreover, compared with the linear theory anlysis, it is of independent of the wavelength of pump wave and the density scale length of plasma.

This paper is arranged as follows: In Sec. 2, we will introduce the nonlinear fluid model and equations. In Sec. 3, benchmark of the code with TPD threshold in unmagnetized plasma is presented. In Sec. 4, some numerical results of the magnetized plasma in electron cyclotron frequency are outlined. Finally, the conclusions are summarized in Sec. 5.

\section{Physical model and equations}

The propagation equations of an electromagnetic wave in plasma produced by the antenna are given by

$$
\begin{aligned}
& \nabla \times \boldsymbol{E}=-\mu_{0} \frac{\partial \boldsymbol{H}}{\partial t} \\
& \nabla \times \boldsymbol{H}=\varepsilon_{0} \frac{\partial \boldsymbol{E}}{\partial t}+\boldsymbol{J}
\end{aligned}
$$

where, $\varepsilon_{0}$ and $\mu_{0}$ are the permittivity and permeability in vacuum, $\boldsymbol{J}$ is the current density in plasma

$$
\boldsymbol{J}=\sum_{\alpha} q_{\alpha} n_{\alpha} \boldsymbol{u}_{\alpha}
$$

The subscript $\alpha$ is the particle species (such as electrons or ions). $q_{\alpha}, n_{\alpha}$ and $\boldsymbol{u}_{\alpha}$ are the charge, plasma density, and velocity, respectively. $n_{\alpha}=n_{\alpha 0}+\delta n_{\alpha}$, where $n_{\alpha, 0}$ is the equilibrium density, and $\delta n_{\alpha}$ 
is the disturbance density of the plasma.

Continuity and moment equations are expressed by

$$
\begin{aligned}
& \frac{\partial n_{\alpha}}{\partial t}+\nabla \cdot\left(n_{\alpha} \boldsymbol{u}\right)=0 \\
& \frac{\partial \boldsymbol{u}_{\alpha}}{\partial t}+\boldsymbol{u}_{\alpha} \cdot \nabla \boldsymbol{u}_{\alpha}=\frac{q_{\alpha}}{m_{\alpha}}\left(\boldsymbol{E}-\nabla \Psi+\frac{\boldsymbol{u}_{\alpha} \times\left(\boldsymbol{B}+\boldsymbol{B}_{0}\right)}{c}\right)-\frac{\gamma_{\alpha} T_{\alpha}}{m_{\alpha}} \frac{1}{n_{\alpha}} \nabla \delta n_{\alpha}
\end{aligned}
$$

where $\gamma_{\alpha}$ is the ratio of specific heats, $\boldsymbol{B}$ is the disturbing magnetic field of electromagnetic wave, and $\boldsymbol{B}_{\boldsymbol{0}}$ is the external static magnetic field, for the unmagnetized plasma, $\boldsymbol{B}_{\boldsymbol{0}}=0, \Psi$ is the wave potential, which can be described by the Poisson's equation

$$
\nabla^{2} \Psi=-e\left(\delta n_{i}-\delta n_{e}\right) / \varepsilon
$$

Equations (1) to (6) comprise a set of coupled and closed equations, and supply to describe the nonlinear interactions between wave and plasma.

\section{Benchmark of the code with TPD threshold in unmagnetized plasma}

The results of TPD's threshold conditions between the analytical and numerical solutions are compared. The analytical threshold condition is from the results of Ref. 11,

$$
\frac{v_{0}^{2}}{v_{e}^{2}} k_{0} L_{n}>3.0
$$

And the parameters (the laser intensity and wavelength, the electron temperature and density scale length) of numerical simulations are shown in Table 1 . Where, $\alpha=0.080 \times L_{\mu m} \sqrt{I_{14}}$ and $\beta=1.41 \times T_{\mathrm{keV}}^{2} /\left(I_{14} \lambda_{\mu}^{2}\right)$ are the two dimensionless coefficients from Ref. 11, another important parameter is TPD threshold $\equiv\left(I_{14} \lambda_{\mu m} L_{\mu m} / T_{\mathrm{keV}}\right) / 59.4013$, and it also can be expressed by threshold $=[3 \alpha /(4 \sqrt{\beta})] / 3.0$, the value of 3.0 is Liu-Rosenbluth's result while the WKB-perturbation's result is 2.756. From the comparison given in Fig. 1, it is not difficult to find out that the simulation results are extremely similar to the theories analytical results.

Table1. Simulation parameters

\begin{tabular}{ccccccc}
\hline \hline $\boldsymbol{\alpha}$ & $\boldsymbol{\beta}$ & Threshold & $\lambda(\mu \mathrm{m})$ & $\boldsymbol{I}_{\boldsymbol{1 4}}\left(\mathrm{W} / \mathrm{cm}^{2}\right)$ & $T(\mathrm{keV})$ & $L(\mu \mathrm{m})$ \\
\hline 0.6581 & 0.025 & 0.99390 & 1.05 & 0.0024 & 0.0065 & 167 \\
2.2590 & 0.25 & 1.3467 & 1.05 & 0.0286 & 0.0712 & 167 \\
4.9501 & 1.0 & 1.4657 & 1.05 & 0.0456 & 0.0975 & 167 \\
7.3391 & 2.0 & 1.5456 & 1.05 & 0.2033 & 0.428 & 167 \\
12.1116 & 5.0 & 1.6149 & 1.05 & 1.4840 & 2.914 & 167 \\
14.9762 & 7.5 & 1.6329 & 1.05 & 3.3389 & 6.684 & 167 \\
17.3621 & 10 & 1.6399 & 1.05 & 6.0248 & 11.978 & 167 \\
\hline \hline
\end{tabular}

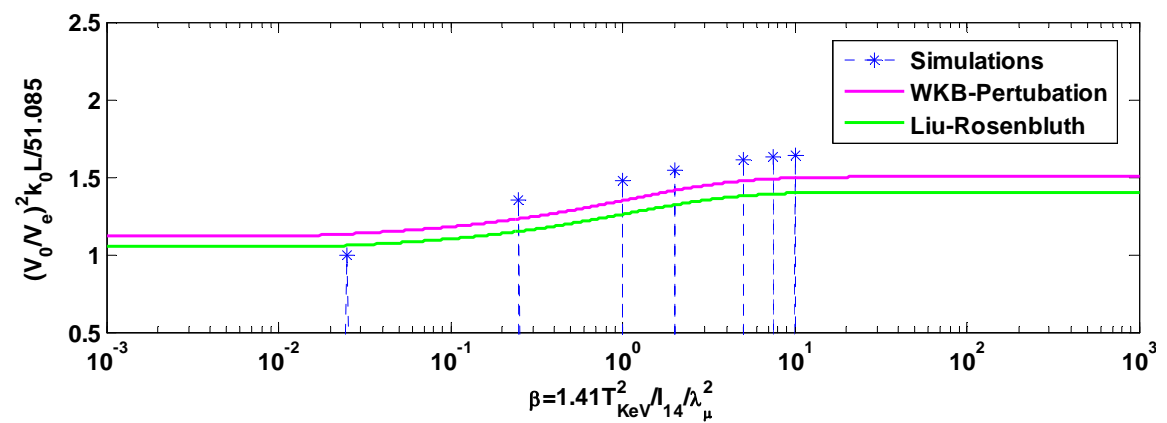

Fig. 1. Compares of the TPD threshold in simulations (Blue stars) and in theory (Pink line: the numerical solution of the Wentzel-Kramers-Brillouin perturbation theory in Ref. 11; Green line: the analytical solution of Liu-Rosenbluth theory in Ref. 11).

\section{Simulations of PDIs in inhomogeneous magnetized plasma}

In Tokamaks, the upper hybrid wave (UHW) is excited when the frequency of $X$ wave equals to the local UHW frequency (EM(Electromagnetic Wave) $\rightarrow$ UHW) [7], named upper hybrid resonance. This is a very normal linear physical process, but if the intensity of the applied electromagnetic 
wave is high enough to reach the threshold condition, the PDIs will take place. Under the scheme of fluid model, there are a few possible decay channels near the upper-hybrid layer. First of all, the produced UHW will decay into a low hybrid wave (LHW) and a sideband UHW [9], called upper-hybrid decay instability (UDI); secondly, the electron cycle wave decays into a LHW and an electromagnetic sideband wave, sometimes this channel is called Raman Scattering of magnetized plasma; thirdly, the electron cyclotron wave decays into LHW and UHW directly. The schematic of the simulation setup is shown in Fig. 2(a), and the background density and external magnetic profiles are given through $\omega_{\mathrm{pe}}$ (electron plasma frequency) and $\omega_{\mathrm{ce}}$ (electron gyro frequency), respectively. For this simulation, the pump frequency, electron and ion temperature, external magnetic field, the density scale length and pump wave intensity are $f_{0}=\omega_{0} /(2 \pi)=60 \mathrm{GHz}$, $T_{e}=4 * T_{i}=160 \mathrm{eV}, B_{0}=1.5 \mathrm{~T}, L_{n}=5.4 \mathrm{~cm}, E_{0}=1.0 \mathrm{e}+5 \mathrm{~V} / \mathrm{m}$. From Fig. 2 (a) we can see that the incident extraordinary wave will resonate with UHW at the upper hybrid resonance position, Fig. 2(b) supply the spatial electrostatic field distribution of upper hybrid resonance, which means that, in the resonance layer, the amplitude of electrostatic field can achieve to a sufficiently larger value.

In order to test whether the first type of PDIs at the upper hybrid layer, we use the parameter profiles of Fig. 2(a) and apply an external electrostatic disturbance at the position where the upper hybrid resonance takes place, and in order to ensure that the threshold condition is similar to the situation of Fig. 2, the amplitude of electrostatic field produced by the external disturbance is almost in accordance with the result of Fig. 2(b). The results are shown in Fig. 3(a) and (b), therefore, it is reasonable to indicate that the strong UDI will arise, even the up-shift cascades of UDI can also be found. In Fig. 3(c), the frequency spectrum of electron static frequency has a very obvious UHW and its sidebands, and together with the local LHW, the three-wave parametric process come into being, combining with the Fig. 3(a) and (b), it can be confirmed that that UDI exists exactly in the upper hybrid layer when the maximal electric field of the incident $X$ wave is about $5 \mathrm{e}+4 \mathrm{~V} / \mathrm{m}$. From the frequency spectrum of magnetic field, the pump $X$ wave is scattered by LHW, but the position of the maximal transverse magnetic field of sidebands is not exactly at the point of upper hybrid resonance, and these sidebands will not be detected outside of plasma area. But it should be noted that, from our results, we can't identify whether the third type of PDIs take place in the upper hybrid resonance layer. In additional, because of these PDIs are directly related to the propagation of wave, the energy absorption and transport process of plasma, even the change of density profile. Therefore, they are of increasing importance and eventually can become dominant with the injected power of antenna launching become higher and higher in the future.
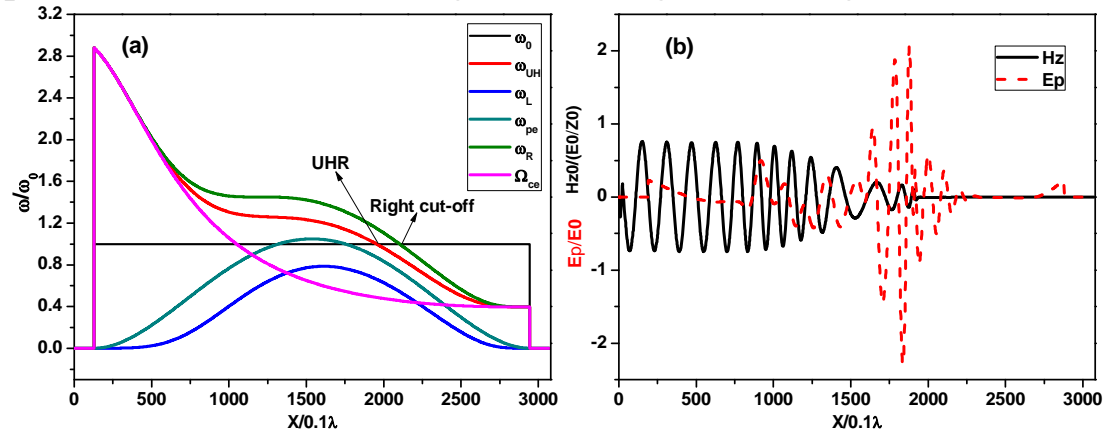

Fig. 2. (a) Schematic of the parameters along $X$ direction during electron cyclotron frequency, where $\omega_{0}, \omega_{\mathrm{UH}}, \omega_{\mathrm{L}}, \omega_{\mathrm{pe}}, \omega_{\mathrm{R}}, \omega_{\mathrm{ce}}$ correspond to these frequencies: incident wave, upper-hybrid resonance, left cut-off, electron plasma, right cut-off, and electron gyro frequency, respectively; (b) Plots of the disturbance magnetic $(\mathrm{Hz})$ and electrostatic $(E p)$ field at $\mathrm{t}=1256 / \omega_{0}$. 

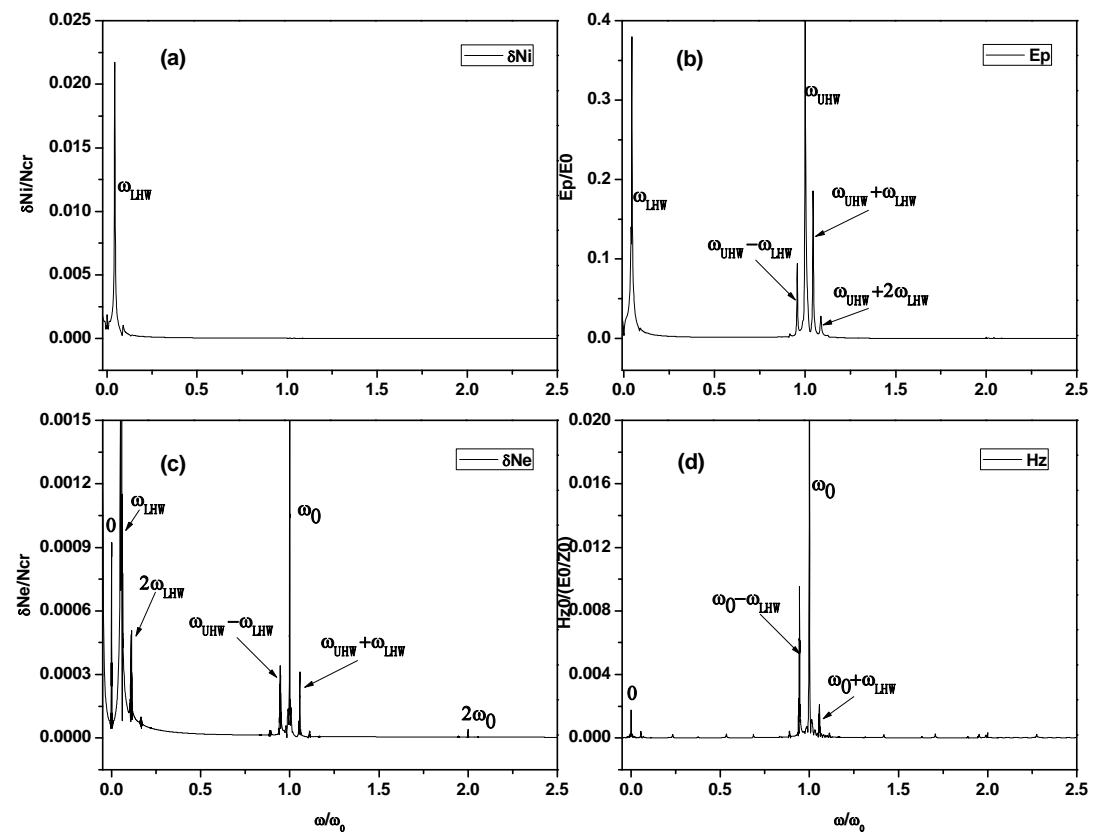

Fig. 3. Spectrum analyses of the normalized components by applying an external electrostatic disturbance at the position of upper hybrid resonance: (a) Electrostatic ion density perturbation and (b) Electrostatic potential field; Spectrum analyses of the normalized components by the incident $X$ pump wave from high field-side:(c) Electron density perturbation and (d) Magnetic-field component of electromagnetic wave.

\section{Summary}

In summary, a full space-time two-fluid plasma simulation model has been developed to improve both nonlinear physics and computation power. Neither in unmagnetized nor in magnetized plasma, there may be various decay channels, and all kinds of possible processes can be obtained self-consistently by using the full space-time method. Moreover, the benchmark of the code with TPD threshold in unmagnetized plasma shows that the full space-time method and the two-fluid code are valid. Furthermore, at the upper hybrid resonance layer of the wave heating of electron cyclotron wave in Tokamak plasma, there are still a few PDIs arising. And if we consider the kinetic linear mode conversion, of which the UHW is converted into electron Bernstein wave, we believe that the PDIs will become richer [9].

\section{Acknowledgements}

This work was financially supported by supported by the National Natural Science Foundation of China under Grant Nos 10990214 and 10775450

\section{References}

[1] M. Porklab: Nucl. Fusion Vol. 18(1978), p. 367

[2] C. S. Liu and V. K. Tripathi: Physics Reports Vol. 130(1986), p. 143

[3] M. N. Rosenbluth: Phys. Rev. Lett. Vol. 29(1972), p. 565

[4] P.Koester and L. Antonelli: Plasma Phys. Control. Fusion Vol. 55(2013), p. 124045

[5] D. W. Forslund, J. M. Kindel, E. L. Lindman, et al.: Phys. Fluids Vol. 18(1975), p. 1017

[6] R. Yan, A. V. Maximov, C. Ren: Phys. Plasmas Vol. 17(2010), p. 052701

[7] A. T. Lin and C. C. Lin: Phys. Rev. Let. Vol. 47(1981), p.98

[8] V. K. Tripathi and C. S. Liu: Phys. Fluids Vol. 25(1982), p. 1388

[9] T. G. Jenkins, T. M. Austin, D. N. Smithe, et al.: Phys. Plasmas, Vol. 20(2013), p. 012116

[10] D. N. Smithe: Phys. Plasmas Vol. 14(2007), p. 056104

[11] A. Simon, R. W. Short, E. A. Williams: Phys. Fluids Vol. 26(1983), p. 3107 